\title{
Lesiones de los ligamentos de los dedos
}

\author{
Alfredo Quintana Guitián
}

HOSPITAL FREMAP. ZARAGOZA

Correspondencia:

alfredo_quintana@fremap.es

$\mathrm{L}$ as lesiones de los ligamentos de los dedos son lesiones frecuentes en la patología de la mano, que habitualmente requieren un tratamiento ortopédico, y que a su aparente banalidad se contrapone la frecuente existencia de secuelas dolorosas o de rigidez articular. Las lesiones cápsulo-ligamentosas digitales predominantes son los esguinces y luxaciones de las articulaciones interfalangicas proximales (IFP) de los dedos largos y de la articulación metacarpofalángica $(\mathrm{MCF})$ del pulgar. A pesar de su frecuencia no son demasiadas las referencias bibliográficas sobre dicha patología. Realizada una búsqueda en el Medline de los últimos cinco años hemos encontrado 32 referencias para el término "finger ligaments injuries" y 12 referencias para "Stener lesión".

Lo fundamental para establecer el tipo de tratamiento, quirúrgico u ortopédico nos vendrá dado por la estabilidad articular. Esta debe ser estudiada tanto de forma pasiva como activa, $y$ será el conjunto de este estudio con las necesidades de la articulación afecta la que determinará el tipo de tratamiento a instaurar.

Como se ha indicado el estudio de la estabilidad articular ha de realizarse tanto en su forma pasiva como en la activa.

Estabilidad pasiva: La exploración de la estabilidad lateral y anteroposterior se realiza de forma bilateral, si es necesario bajo anestesia local, comparando la articulación afecta con la contralateral.

Estabilidad activa: Capacidad del paciente para repetir una subluxación o luxación. El paciente mueve el dedo en su arco de movilidad habitual, si completa el movimiento sin desplazamiento anómalo el dedo es estable. En caso de redesplazamiento estaremos ante una lesión inestable.

\section{LESIONES MCF DE LOS DEDOS LARGOS:}

\section{RECUERDO ANATÓMICO ${ }^{1-4}$}

Hay una serie de características anatómicas que debemos valorar en cuanto a la estabilidad de estas articulaciones:

$1^{\circ}$ La placa volar (PV) es más gruesa y fuerte en la inserción en la falange que en el metacarpiano.

$2^{\circ}$ La placa volar (PV) se encuentra reforzada por el ligamento intermetacarpiano transverso profundo (LIT) y la vaina de los flexores (VF) (Figura 1).

$3^{\circ}$ Los ligamentos laterales (LL) se encuentran más tensos en flexión que en extensión (Figura 2).

$4^{\circ}$ La articulación es más estable en flexión, permitiendo cierto grado de abducción-aducción en extensión.

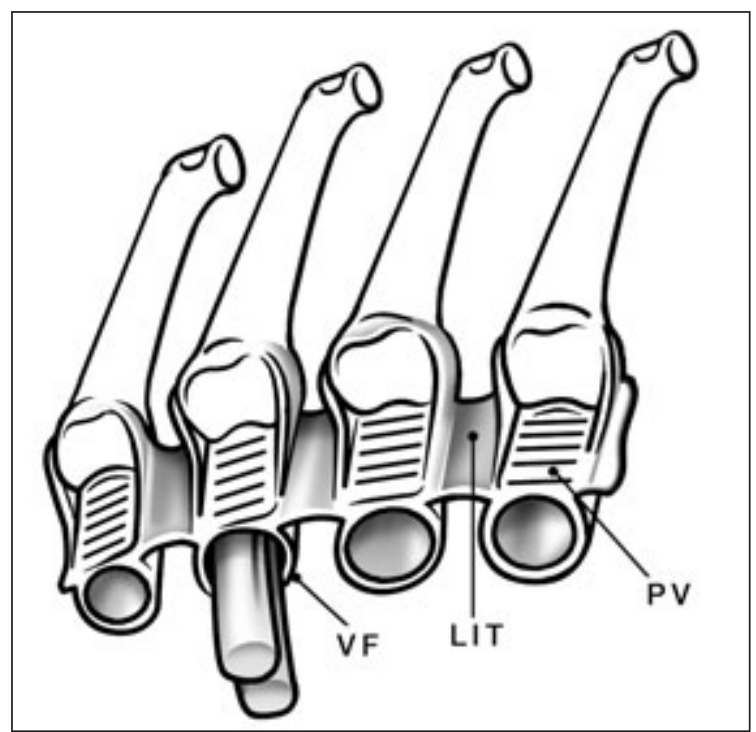

Figura 1: Esquema anatómico de la placa volar de la MCF de los dedos largos. 


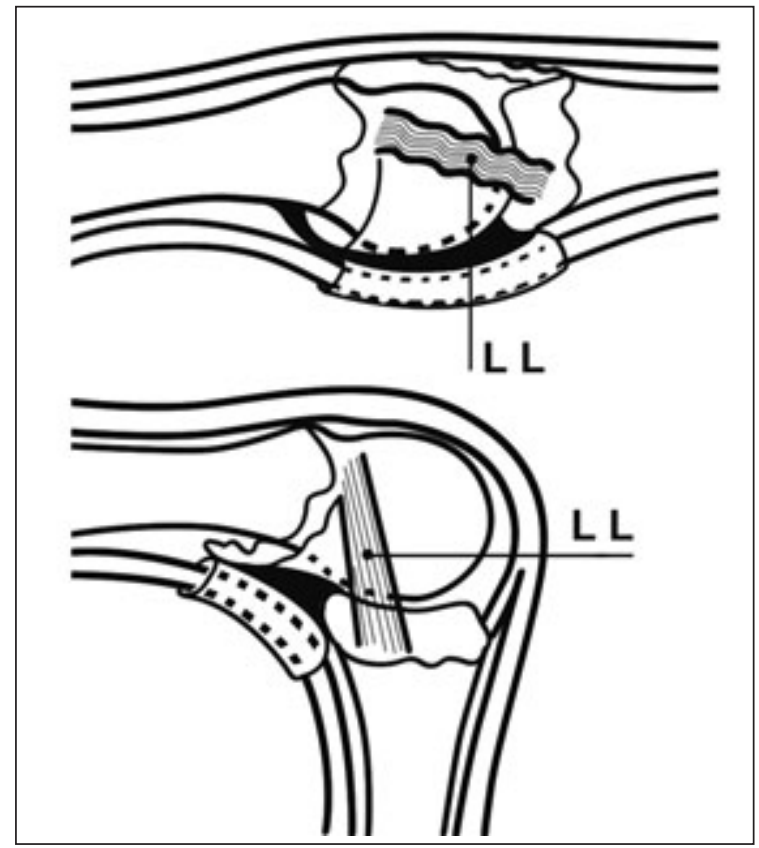

Figura 2: Esquema anatómico de la articulación MCF de los dedos largos: a. Los ligamentos laterales se relajan en extensión; $b$. Los ligamentos laterales se tensan en flexión

\section{ESGUINCES}

Son lesiones que generalmente obedecen a un mecanismo de hiperextensión y desviación cubital, afectando sobre todo a los tres dedos cubitales. La rotura de un ligamento a este nivel puede producirse de forma aislada o con un arrancamiento óseo, las roturas del ligamento cubital son muy raras dándose con mayor frecuencia la rotura del colateral radial; son lesiones que frecuentemente son infravaloradas y que en ocasiones originan problemas residuales ${ }^{5}$. El arrancamiento habitualmente se produce en la base de la falange. También se han descrito casos de rotura del ligamento intermetacarpiano ${ }^{6} \mathrm{o}$ de la placa volar ${ }^{7}$.

En cuanto al tratamiento es generalmente de tipo ortopédico ${ }^{8}$ mediante una inmovilización de unas tres semanas manteniendo la articulación en flexión de unos $35^{\circ}$. En aquellos casos en que hay una marcada inestabilidad, afectando sobre todo al índice o al quinto dedo nos podemos plantear una reparación quirúrgica mediante reinserción del ligamento. Asimismo hay que plantearse el tratamiento quirúrgico en aquellos casos en que existe un arrancamiento óseo que afecte a la superficie articular en más de un $25 \%$, o en aquellos casos en los que hay una separación de más de $3 \mathrm{~mm}^{4,9-13}$.

\section{LUXACIONES}

La luxación habitual es la dorsal en la cual la falange se sitúa en el dorso del metacarpiano; son lesiones poco frecuentes y que generalmente afectan al índice y al quinto dedo ${ }^{4,14-17}$. En caso de que sólo exista una hiperextensión de la falange sobre la cabeza del metacarpiano, manteniendo contacto entre las superficies articulares, nos encontramos con la subluxación, en la cual la placa palmar permanece en la cara anterior de la cabeza del metacarpiano sin interponerse en la articulación.

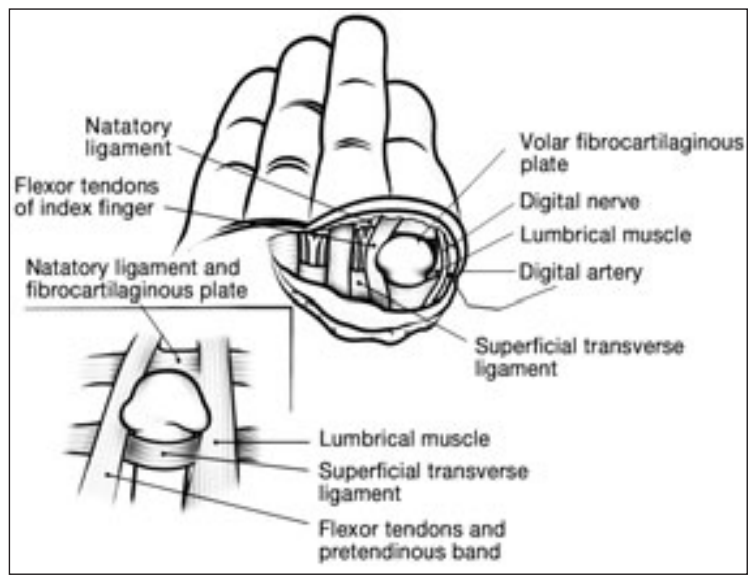

Figura 3: Esquema de Kaplan explicando la irreductibilidad de la luxación MCF ${ }^{18}$.

Fue Kaplan ${ }^{18}$ en 1957 quién describió la situación anatómica de esta lesión que hace que en muchas ocasiones sea irreductible de forma ortopédica, requiriendo una liberación quirúrgica. Como podemos ver en el dibujo original de Kaplan (Figura 3) hay varias estructuras que impiden la reducción de la articulación; por un lado la placa volar (PV) que se desprende de su inserción proximal y se interpone en la articulación, por otro los tendones de los dedos índice o meñique que se sitúan a los lados del cuello del metacarpiano y que forman un lazo (LT) a su alrededor impidiendo la reducción. (Figura 4). En el índice en el lado radial del metacarpiano se sitúa el lumbricalis y en el lado cubital los tendones del flexor digitorum profundus y del superficialis. En el meñique el abductor digiti minimi y el flexor digiti minimi se situán en el lado cubital, 


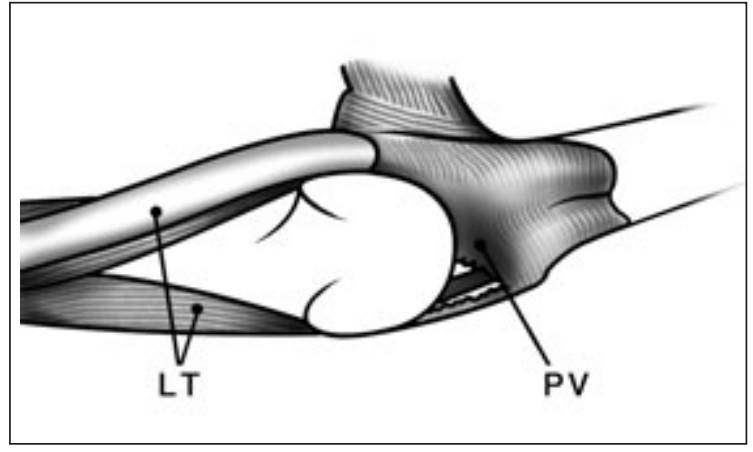

Figura 4: Detalle de la interposición de la placa volar y el “lazo" tendinoso alrededor del cuello del metacarpiano ${ }^{1}$.

mientras que por el lado radial el lazo lo forman los flexori digitorum y el lumbricalis.

En aquellos casos en que la placa volar se ha desprendido del metacarpiano, pero no se ha llegado a interponer en la articulación (subluxación), generalmente nos encontraremos con la falange en una hiperextensión de $60^{\circ}$ a $80^{\circ}$, siendo factible el realizar una reducción ortopédica mediante maniobra de flexión de la falange mientras se mantiene presionada sobre la cabeza del metacarpiano. Una vez reducida se mantendrá inmovilizada impidiendo la extensión unas dos semanas

El abordaje quirúrgico en aquellos casos en los que es imposible la reducción ortopédica fue iniciado por Farabeuf ${ }^{19}$ en el siglo XIX. En la actualidad se tiende a realizar una incisión volar $\mathrm{y}$ tras seccionar la polea $\mathrm{A} 1$, lo que permite una relajación de la tensión del tendón flexor y eliminar el cerco alrededor de la cabeza del metacarpiano, se manipula la falange y la placa volar para que vuelvan a su posición; tras suturar la piel se mantiene la articulación en flexión de $30^{\circ}$ durante dos semanas bloqueando posteriormente la hiperextensión.

Aunque están descritas luxaciones volares $^{14,20-22}$ son bastante raras y generalmente se logra la reducción cerrada de forma satisfactoria. En caso de no lograrse se debe realizar una reducción abierta a través de un abordaje dorsal.

\section{LESIONES CRÓNICAS-SECUELAS}

Las lesiones crónicas de las articulaciones MCF son raras, siendo la más común la desviación cubital del quinto dedo por rotura del liga- mento radial y de las conexiones intertendinosas que favorece la desviación cubital del extensor, así como por la acción del abductor digiti minimi ${ }^{23}$.

\section{LESIONES IFP DE LOS DEDOS LARGOS}

\section{RECUERDO ANATÓMICO1,2,4}

La articulación IFP presenta como elementos estabilizadores (Figura 5) :

a. los ligamentos colaterales (LC), que se insertan en la base de la falange

b. los ligamentos colaterales accesorios (LCA) que lo hacen en la placa palmar

c. la placa palmar (PV), más densa en la base de la segunda falange

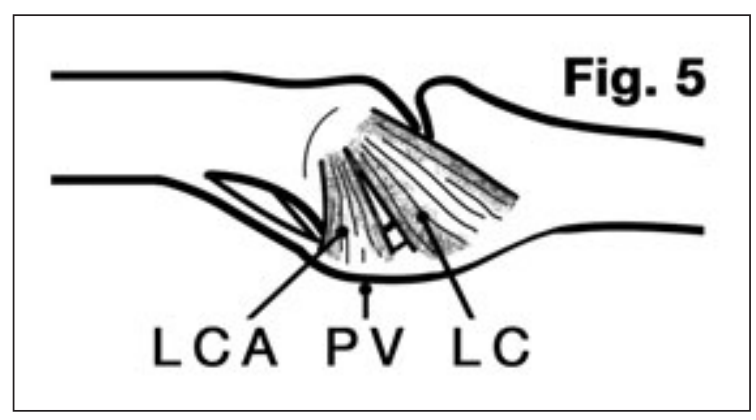

Figura 5: Esquema anatómico de los ligamentos de la IFP de los dedos.

\section{EVALUACIÓN CLÍNICA}

Para determinar el tipo de lesión nos hará falta un estudio radiológico centrado en la articulación en proyección anteroposterior y lateral, así como un estudio de la estabilidad articular, tanto en forma activa como pasiva.

\section{ESGUINCES}

1. Esguinces de los ligamentos laterales: Se producen sobre todo en el $4^{\circ}$ y $5^{\circ}$ dedos por un choque lateral con el dedo en extensión. Clínicamente hay edema y posible hematoma, siendo poco manifiesta la laxitud articular; ocasionalmente puede apreciarse en el estudio radiológico un pequeño arrancamiento óseo en la base de la segunda falange. El tratamiento general es una sindactilia durante 15 días. 
2. Esguince de la placa palmar: Es el resultado de un mecanismo de hiperextensión, produciéndose habitualmente un hematoma en la cara volar de la articulación, así como edema. Se produce un arrancamiento parcial o total de la placa palmar en la segunda falange, que en ocasiones se acompaña de un pequeño arrancamiento óseo. La cicatrización de la placa palmar suele tender a la retracción, por lo que uno de los riesgos de esta lesión es la rigidez articular. El tratamiento debe encaminarse a la movilización precoz en lugar de la inmovilización, mediante el uso de una sindactilia o una férula que permita la flexión e impida la hiperextensión durante 15 días.

\section{LUXACIONES}

Las luxaciones IFP más frecuentes son las dorsales, en las cuales la falange media se coloca dorsalmente respecto a la proximal; otros tipos de luxaciones menos frecuentes son las laterales y las volares.

\section{Luxación dorsal:}

Son lesiones que se producen por un mecanismo de hiperextensión y compresión longitudinal de la falange media. Anatomopatológicamente las lesiones que se producen son la rotura de la placa volar generalmente a nivel distal, rotura parcial o total del ligamento colateral sin desinserción, y en algunas ocasiones un arrancamiento óseo.

Lo fundamental en cuanto al tratamiento es valorar la estabilidad articular y en caso de existir un arrancamiento óseo el tamaño del mismo. Es necesario realizar un estudio radiológico centrado en la articulación en posición posteroanterior y lateral, así como valorar la estabilidad articular de forma pasiva y activa.

Podemos diferenciar este tipo de luxaciones en dos grandes apartados, las lesiones estables, cuyo tratamiento será fundamentalmente ortopédico y las inestables cuyo tratamiento será quirúrgico.

\section{A. Lesiones estables:}

1. Subluxación (hiperextensión): Se produce una avulsión parcial o completa de la placa volar en la base de la falange, siendo posible una rotura parcial longitudinal del ligamento colateral; ocasionalmente hay un pequeño fragmento óseo avulsionado. La articulación permanece congruente.
2. Luxación (Figura 6): Además de la avulsión de la placa volar (PV) hay una lesión bilateral de los ligamentos colaterales (LC). No hay contacto entre las superficies articulares óseas, disponiéndose la falange media dorsal en relación a la proximal.

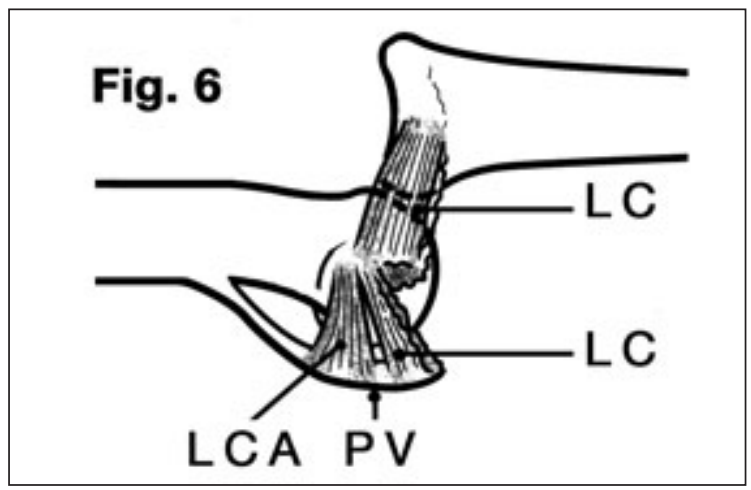

Figura 6: Esquema de la lesión de los ligamentos de la IFP en la luxación.

3. Fractura luxación (Figura 7 A): En ella en lugar de arrancarse la placa volar (PV) lo hace un fragmento óseo (FO) de menos del $40 \%$ de la base de la falange. El ligamento colateral (LC) al menos parcialmente en su zona más dorsal permanece unido a la falange media, lo que hace estable la articulación.

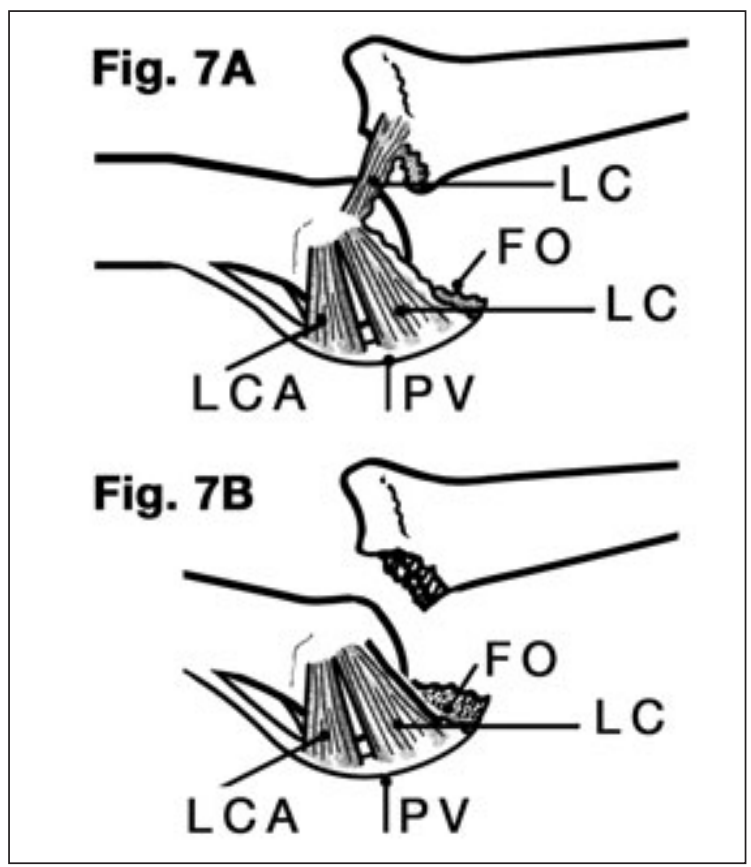

Figura 7: Esquema de las lesiones en la fractura luxación de la IFP. 
En cuanto al tratamiento de este tipo de lesiones es habitualmente de tipo ortopédico ${ }^{2,4,23}$, siendo escasos los casos que precisan tratamiento quirúrgico ${ }^{24,25}$. Una vez reducida la articulación se mantendrá una férula de inmovilización con la articulación flexionada unos $25^{\circ}$, variando el tiempo de inmovilización en dependencia de la lesión, pudiendo establecerse de forma general los siguientes periodos:

Subluxación: Una semana de inmovilización.

Luxación: Dos semanas de inmovilización.

Fractura-luxación: Tres semanas de inmovilización.

\section{B. Lesiones inestables:}

1. Fractura luxación (Figura 7 B): Si el fragmento (FO) avulsionado de la falange es mayor del $40 \%$ tanto la placa volar como la mayor parte del la inserción del ligamento colateral queda unido al fragmento avulsionado, siendo difícil de reducir la luxación, y en caso de conseguirlo suele ser lesiones muy inestables reproduciéndose la luxación ${ }^{23}$.

2. El tratamiento de estas lesiones es complejo y habitualmente de naturaleza quirúrgica, no obteniéndose habitualmente unos resultados excelentes, pudiendo utilizarse los siguientes procedimientos $^{2,4,23,26-31}$ :

a. Tracción esquelética:

b. Bloqueo articular con aguja de Kirschner

c. Reducción abierta y osteosíntesis

d. Artroplastia de placa volar.

\section{Luxación lateral:}

Una fuerza lateral puede producir el arrancamiento del ligamento colateral, así como una avulsión parcial o total de la placa volar. Clínicamente comprobaremos la estabilidad articular con el dedo en extensión, en caso de que exista una desviación lateral superior a $20^{\circ}$ hemos de presumir la existencia de una rotura del ligamento lateral.

El tratamiento suele ser de tipo ortopédico pudiéndose optar bien por una inmovilización con una férula o bien mediante el uso de una sindactilia. El tratamiento quirúrgico en nuestra opinión debe guardarse para aquellos raros casos en los que la reducción es imposible o hay reluxación con los movimientos activos, aunque hace años algunos autores planteaban el trata- miento quirúrgico de entrada ${ }^{32,33}$; este tratamiento también ha sido defendido por algunos autores en deportistas. Asimismo se debe plantear la solución quirúrgica en aquellos casos en los que exista una inestabilidad de tipo crónico.

\section{Luxación palmar:}

Son lesiones poco frecuentes, siendo su causa una fuerza de compresión longitudinal rotatoria sobre una falange media en semiflexión. Las estructuras lesionadas son la placa volar que se avulsiona parcialmente, un ligamento colateral y suele conllevar lesión del aparato extensor. Está última lesión puede ser de dos tipos, o bien rotura de la bandeleta central (luxación volar), o bien interposición de la cabeza de la falange proximal entre la bandeleta central (BC) y una de las laterales del extensor (BL) (luxación rotatoria volar) (Figura 8).

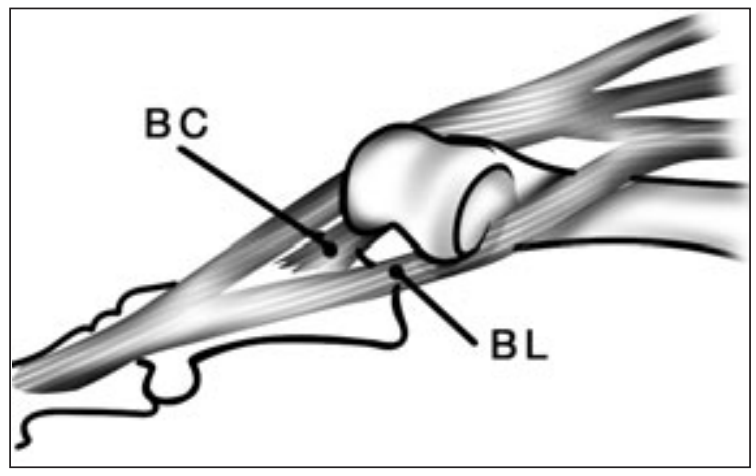

Figura 8: Esquema de la luxación rotatoria volar ${ }^{2}$.

En cuanto al tratamiento la luxación volar pura suele ser fácil de reducir, y una vez logrado debemos mantener la articulación en extensión durante 4-6 semanas para tratar la lesión en ojal del extensor.

Los casos de luxación rotatoria son más difíciles de reducir, debiendo probarse con una tracción y rotación de la falange media manteniendo la MCF en flexión para relajar el aparato extensor. Una vez reducida se debe valorar la función del aparto extensor, en caso de presuponer una rotura de la bandeleta central o lateral (déficit de extensión) se deberá inmovilizar durante seis semanas en extensión.

En aquellos casos en los que no se puede lograr una reducción de la luxación ${ }^{34}$, o que se trate de una fractura luxación con fragmento de más del $40 \%$ será necesario el tratamiento quirúrgico. 


\section{Secuelas de las luxaciones}

1. Fibrosis postraumática y rigidez articular: El edema postraumático de la articulación suele conllevar una fibrosis y ésta a una cicatriz del ligamento; hasta el desarrollo final del proceso puede transcurrir un año. Para su prevención se debe establecer un programa rehabilitador y uso de férulas para mejorar la movilidad. En caso de que se establezca una rigidez en dependencia de la limitación deberemos valorar la posibilidad de realizar un tratamiento quirúrgico. Este consistirá en resecar los ligamentos colaterales ${ }^{32}$, habitualmente primero el cubital y si no cede el radial; si persiste la imposibilidad de la extensión habrá que proceder a liberar la placa volar en su inserción distal.

2. Hiperextensión articular: Tras una luxación con una rotura de la placa volar esta puede quedar alargada o desinsertada, lo que originará la posibilidad de que se produzca una hiperextensión articular. El tratamiento consistirá en suturar o reinsertar la placa volar. Otro posible tratamiento es realizar una estabilización articular mediante el uso de una tenodesis, bien con una o ambas bandeletas del flexor digitorum superficialis, $\mathrm{o}$ con una bandeleta tomada del extensor ${ }^{35,36}$.

3. Inestabilidad lateral: Es una secuela rara y para su tratamiento requeriría la sutura del ligamento lateral

4. Dolor e inflamación articular.

\section{LESIONES IFD DE LOS DEDOS LARGOS}

La articulación IFD de los dedos presenta como las IFP elementos estabilizadores como los ligamentos laterales y la placa palmar, además las inserciones tendinosas ayudan a dar una mayor estabilidad articular, por lo que no es muy frecuente su patología.

Las luxaciones suelen ser dorsales o laterales, y frecuentemente asocian herida cutánea. Habitualmente son fáciles de reducir siendo además estables, por lo que suele bastar con tratamiento ortopédico e inmovilización en ligera flexión $\left(15^{\circ}\right)$ durante dos o tres semanas. Se han descrito casos de luxaciones volares que requieren inmovilización en extensión durante seis semanas.
Asimismo están descritos casos de irreductibilidad ${ }^{37}$ por interposición de la placa volar o del tendón flexor, así como por la interposición de un fragmento óseo avulsionado, así como casos de luxación a nivel de IFP e IFD ${ }^{38}$.

En ocasiones queda como secuelas una menor movilidad articular por fibrosis de la capsula y de los ligamentos, y en otras ocasiones dolores residuales que son difíciles de tratar, siendo necesario en ocasiones tener que realizar la artrodesis articular con distinto grado de flexión en dependencia del dedo afecto.

\section{LESIONES MCF DEL PULGAR}

\section{RECUERDO ANATÓMICO²,439}

Las estructuras estabilizadoras de la articulación MF del pulgar son esencialmente (Figura 9 y 10) :

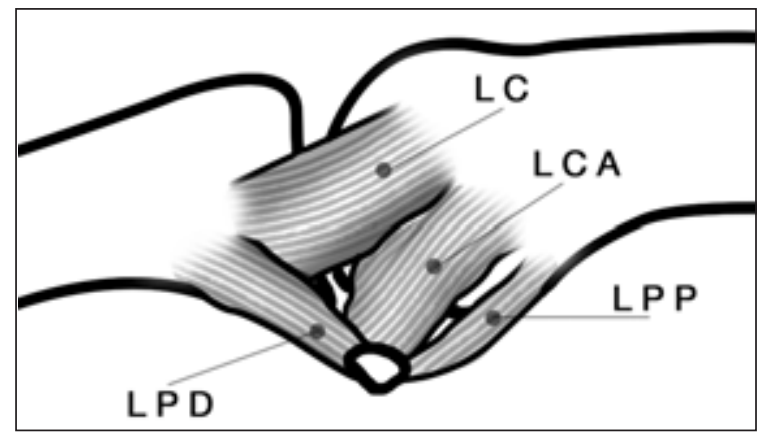

Figura 9: Esquema del sistema cápsulo ligamentoso de la articulación MCF del pulgar. Cara radial ${ }^{2}$.

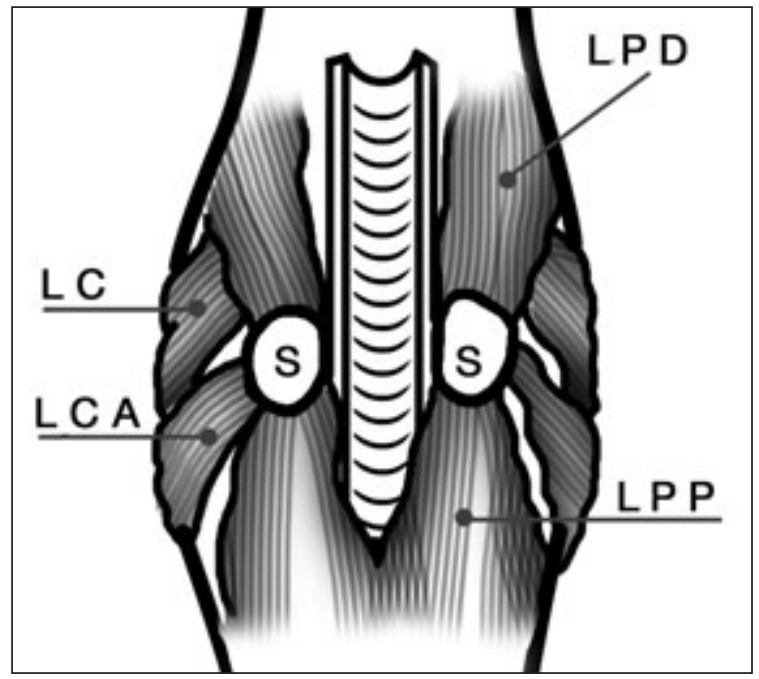

Figura 10: Esquema del sistema cápsulo ligamentoso de la articulación MCF del pulgar. Cara palmar ${ }^{2}$. 
1. Ligamento lateral (LC), el cual se dirige desde la cabeza del metacarpiano hasta la base de la falange proximal, tensándose con la flexión articular.

2. Ligamento lateral accesorio (LCA) se dirigen desde la cabeza del metacarpiano al sesamoideo $(\mathrm{S})$

3. Placa palmar : Dividida en una zona proximal (LPP) y otra distal (LPD) por los sesamoideos (S)

4. Elementos tendinosos (flexor pollicis brevis $\mathrm{y}$ adductor pollicis) que se insertan en los sesamoideos y que ayudan a dar una estabilidad activa a la articulación.

\section{ESGUINCES}

\section{TIPO DE LESIONES}

1. Lesión por hiperaducción: Se lesiona el ligamento colateral radial. Es menos frecuente que la lesión del ligamento cubital en una proporción de 1/10. El arrancamiento generalmente se produce en su inserción proximal, aunque puede producirse en cualquier otro sitio; en ocasiones se aprecia un arrancamiento óseo de la falange. El ligamento nunca pasa por encima de la aponeurosis del abductor pollicis brevis, lo que facilita el tratamiento ortopédico ${ }^{40}$.

2. Lesión por hiperextensión: La hiperextensión articular irá afectando progresivamente a distintas estructuras hasta poder originar la luxación articular. En primer lugar se rompe el ligamento palmar proximal (refuerzo de la placa volar), lo que permite una hiperextensión de unos $20^{\circ}$; si persiste la fuerza la siguiente estructura lesionada será el ligamento colateral accesorio, lo que permite que la superficie palmar de la base de la falange se coloque a nivel de la superficie dorsal del metacarpiano.

3. Lesión por hiperabducción: Se lesiona el ligamento cubital, habitualmente en el extremo distal del ligamento. El aductor pollicis además de sus inserciones en la primera falange y en el sesamoideo interno, presenta una expansión fibrosa (aponeurosis) (AA) que llega hasta el extensor, si la MCF está flexionada, la aponeurosis del adductor po- llicis se desplaza distalmente y al volver el dedo a su sitio el ligamento colateral cubital (LCC) queda por encima de dicha aponeurosis siendo irreductible ortopédicamente, es la denominada lesión de Stener ${ }^{41}$ que marcara el tratamiento a aplicar (Figura 11). Con cierta frecuencia se produce un arrancamiento en la base de la falange, pudiendo apreciarse o no separación de su inserción original. Se han descrito casos de doble lesión, apreciándose un arrancamiento óseo sin desviación (fractura por cizallamiento de la base de la falange), existiendo asimismo una avulsión del ligamento de la falange (doble lesión), lo que puede llevar a instaurar un tratamiento ortopédico en lugar de la reparación quirúrgica pertinente.

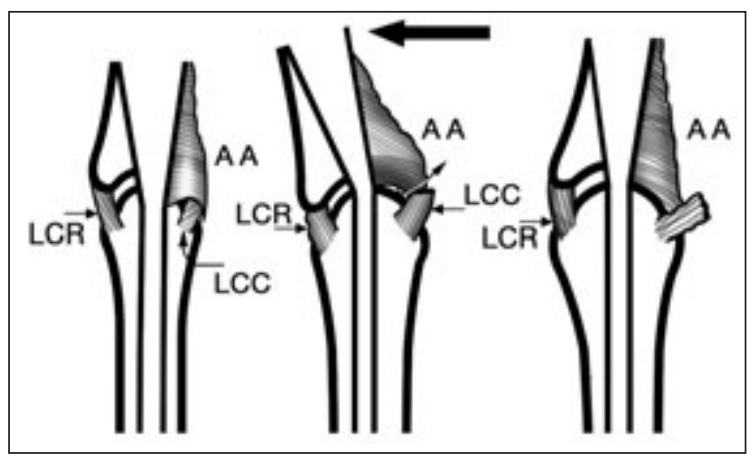

Figura 11: Esquema de la lesión de Stener.

\section{DIAGNÓSTICO}

El diagnóstico de este tipo de lesiones viene predeterminado sobre todo por la clínica ${ }^{42}$. En primer lugar el paciente refiere el antecedente del traumatismo, presentando dolor difuso en la articulación, junto con edema y posible hematoma, así como incapacidad funcional.

Antes de proceder a examinar la laxitud articular debemos realizar un estudio radiográfico simple en dos proyecciones para visualizar la existencia de algún arrancamiento óseo sin desplazamiento, que no haga aconsejable el realizar maniobras para valorar la estabilidad articular.

Seguidamente pasaremos a valorar la estabilidad articular, teniendo en cuenta que el ligamento colateral es el responsable de la estabilidad articular con el dedo flexionado, mientras que la placa palmar y el ligamento colateral accesorio lo son en la extensión ${ }^{43}$. 


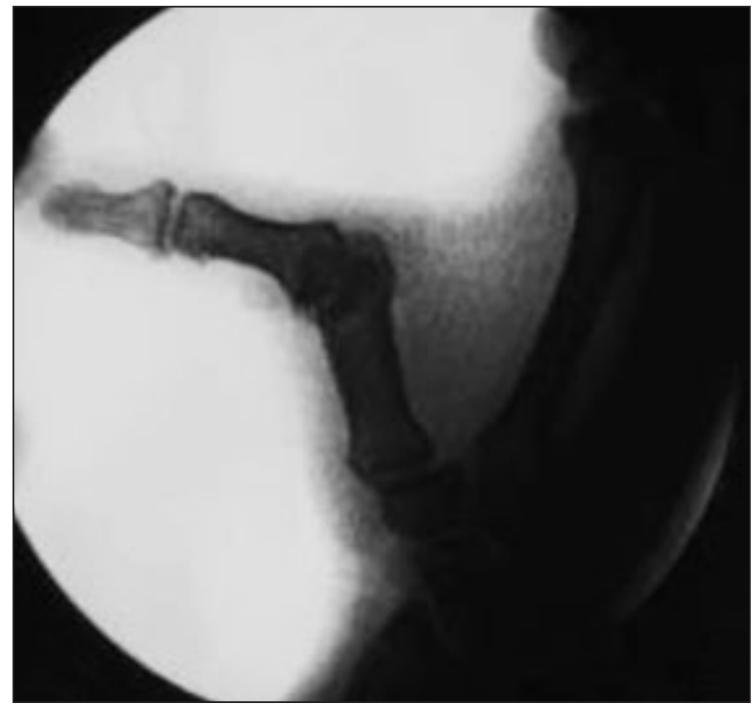

Figura 12: Estudio radiológico mostrando la rotura del ligamento cubital de la MCF del pulgar.

Pasaremos entonces a realizar un estudio radiológico en posición de stress articular; consideraremos la existencia de una rotura del ligamento si con la articulación flexionada $25^{\circ}$ se produce una apertura articular de $30^{\circ}-40^{\circ}$, o un aumento de unos $15^{\circ}$ en relación al dedo contralateral; dicho estudio puede ser conveniente realizarlo bajo anestesia

El estudio con otro tipo de medios de diagnóstico como la ecografía o la RM no suele ser necesario para establecer el diagnóstico y tratamiento de estas lesiones, aunque es defendido por varios autores ${ }^{45-48}$.

\section{TRATAMIENTO}

Las lesiones en las que no se ha producido una rotura completa del ligamento, sin claros signos de inestabilidad se tratarán de forma ortopédica mediante escayolado de 3-4 semanas.

En cuanto a las roturas del ligamento radial el tratamiento varía desde los autores que indican que es suficiente el tratamiento ortopédico a aquellos que se inclinan por la reconstrucción quirúrgica del ligamento. En este caso el tratamiento consistirá en la sutura del ligamento o en caso de estar desinsertado la reinserción con un anclaje. En los casos en los que exista una inestabilidad crónica puede ser necesario la reconstrucción del ligamento a través de una plastia tendinosa ${ }^{49}$.
Las lesiones por hiperextensión con afectación de la placa volar suelen requerir únicamente tratamiento ortopédico manteniendo la articulación en flexión de $15^{\circ}$ unas dos o tres semanas. En caso de que exista una hiperextensión sintomática se puede realizar una reinserción de la placa volar.

En las lesiones completas del ligamento cubital la mayoría de los autores son partidarios del tratamiento quirúrgico ${ }^{2,4}$. Aunque teóricamente si el ligamento se mantiene por debajo de la aponeurosis del adductor pollicis puede llegar a cicatrizar en buena posición, la posibilidad de que no sea así hace inclinar la balanza hacia el ese tipo de tratamiento. No obstante hay autores que recomiendan realizar siempre un tratamiento ortopédico, mientras que otros indican el tratamiento quirúrgico únicamente ante la sospecha de una lesión de Stener (masa palpable en cabeza metacarpiano).

En aquellos casos en los que se ha producido un arrancamiento óseo este será el que nos marcara la pauta a seguir. Si el fragmento no está desplazado y su tamaño es menos del $20 \%$ de la superficie articular se puede optar por un tratamiento ortopédico, sobre todo en caso de que la inestabilidad no sea muy manifiesta; en caso de optar por un tratamiento quirúrgico es factible el resecar el fragmento óseo y reinsertar el ligamento en el defecto óseo mediante un anclaje. En caso de que el fragmento sea mayor deberá ser repuesto en su sitio y fijado bien mediante tornillos o agujas de Kirschner ${ }^{50}$.

Si la lesión afecta exclusivamente al ligamento, éste puede haberse roto en su parte media, procediéndose a la sutura de los extremos con un hilo de 4/0; o haberse producido la avulsión de su inserción ósea, en cuyo caso habrá que realizar su reinserción mediante un pull-out o un anclaje $\mathrm{e}^{51,52}$.

En algunas ocasiones se produce una inestabilidad crónica del ligamento cubital, bien por falta de tratamiento adecuado de una lesión aguda o por distensión progresiva del ligamento (pulgar del guardabosques) ${ }^{53}$; esto conlleva una dificultad para realizar la pinza tanto con el pulpejo como la lateral, siendo preciso el realizar un tratamiento para mejorar la estabilidad. Hay varias opciones para intentar dicha estabilización: 
1. Si la lesión no es muy antigua entre 6 y 12 semanas se puede intentar la reconstrucción del ligamento con sus restos los cuales suelen estar retraídos; para ello ha de ser posible el individualizar el ligamento y movilizarlo para que obtenga su longitud fisiológica y reanclarlo en su posición original ${ }^{54}$. Algunos autores refuerzan este tejido con una expansión del adductor pollicis.

2. Neviaser ${ }^{55}$ en 1971 describió el adelantamiento del tendón del adductor pollicis.

3. Plastias tendinosas: Se han descrito distintos procedimientos para sustituir el ligamento cubital por una plastia tendinosa ${ }^{56-59}$, principalmente se han utilizado el extensor pollicis brevis o el palmaris longus, así como procedimientos más complejos como injertos osteoperiósticos ${ }^{60,61}$. Asimismo se han descrito distintas localizaciones para los túneles en falange y metacarpiano ${ }^{62}$.

4. Artrodesis articular: En aquellos casos que fallen los procedimientos indicados y persista una marcada inestabilidad articular se puede plantear la necesidad de realizar este procedimiento.

\section{LUXACIONES}

Las luxaciones de la articulación MCF del pulgar son en su mayoría dorsales, siendo mucho menos frecuentes las palmares.

1. Luxación dorsal: Fue descrita por Farabeuf en $1876^{19}$, se produce por sufrir un traumatismo con el dedo en hiperextensión. Generalmente se produce una rotura proximal de la placa volar y de la cápsula articular, permaneciendo intactos los ligamentos laterales principales ${ }^{63}$; también puede producirse la rotura a nivel de los sesamoideos o más distalmente ${ }^{4}$. En un primer momento se produce una hiperextensión de la MCF, arrastrando la primera falange al conjunto fibrocartílago-sesamoideo por detrás de la cabeza del metacarpiano. La lesión característica de la luxación es la introducción de la cabeza del metacarpiano en un ojal formado por dentro por el flexor pollicis longus y el adductor pollicis y por fuera por el flexor pollicis brevis Podemos distinguir tres tipos de luxaciones: a. Luxación simple incompleta: La base de la falange se presenta en posición de subluxación en la mitad posterior de la cabeza del metatarsiano; persistiendo el contacto articular entre la base de la falange y la cabeza metatarsiana; la cincha sesamoidea ha franqueado el reborde articular anterior de la cabeza del metatarsiano. La reducción es simple.

b. Luxación simple completa (Figura 13) : Clínicamente es fácil de reconocer por presentar una deformidad en hipertensión, con el denominado pulgar en $\mathrm{Z}$, en el cual la falange primera está en hiperextensión y la falange distal en flexión; el diagnóstico se confirma con el estudio radiográfico. En éste la base de la primera falange se encuentra en posición vertical por detrás de la cabeza del metacarpiano; la superficie cartilaginosa de los sesamoideos se apoya sobre la cabeza del metacarpiano, la cincha del sesamoideo permanece por delante del metacarpiano. El tratamiento es eminentemente ortopédico, debiéndose evitar la tracción longitudinal de la falange, que puede convertir la luxación en compleja; manteniendo la falange en ángulo recto respecto al metacarpiano se empuja la base para que se deslice sobre la cabeza del metacarpiano; conviene mantener el metacarpiano en posición de flexión y aducción pues esa posición relaja el "lazo" formado entre el

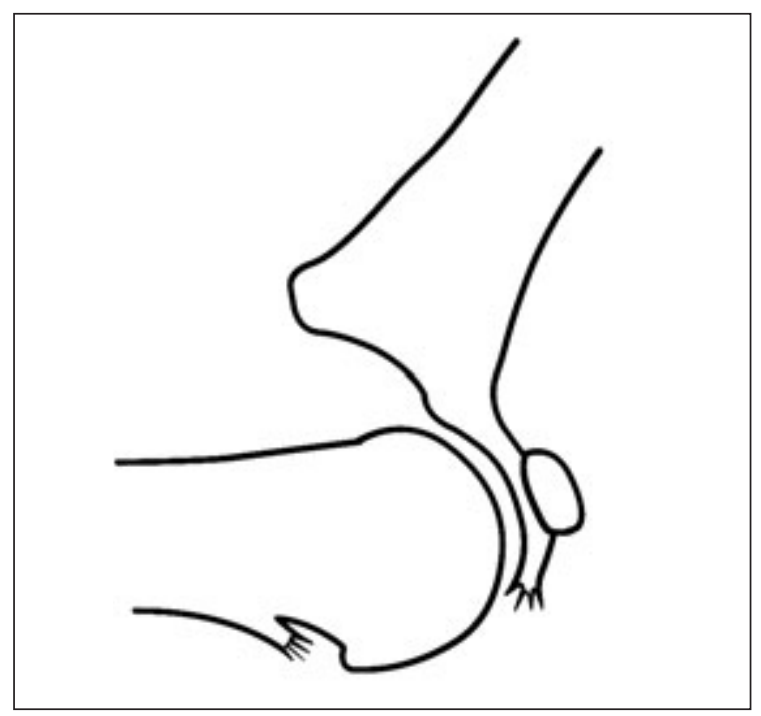

Figura 13: Esquema de la luxación simple completa de la MCF del pulgar. 
flexor pollicis longus y la musculatura intrínseca.

c. Luxación compleja (Figura 14) : Generalmente se produce por maniobras de reducción de la luxación simple completa. Clínicamente la falange muestra un paralelismo en relación al metacarpiano. Muy indicativa de este tipo de lesión es apreciar en el estudio radiográfico la situación de los sesamoideos en posición dorsal al cuello del metacarpiano. La placa volar, los sesamoideos o el tendón del flexor pollicis longus pueden estar atrapados en la articulación impidiendo la reducción ${ }^{4,64,65}$. Se debe intentar la reducción ortopédica una vez, en caso de resultar insatisfactoria será precisa la reducción quirúrgica. El abordaje puede ser dorsal o volar. Se debe revisar la placa volar, y en caso de que esté desprendida de la falange proceder a su reinserción; asimismo habrá que valorar el estado de los ligamentos laterales que habitualmente son estables. Procederemos posteriormente a inmovilizar entre dos y cuatro semanas en dependencia de la estabilidad articular; si es estable es suficiente con dos semanas y posteriormente bloquear la extensión con una férula a $-20^{\circ}$ durante otras dos semanas.

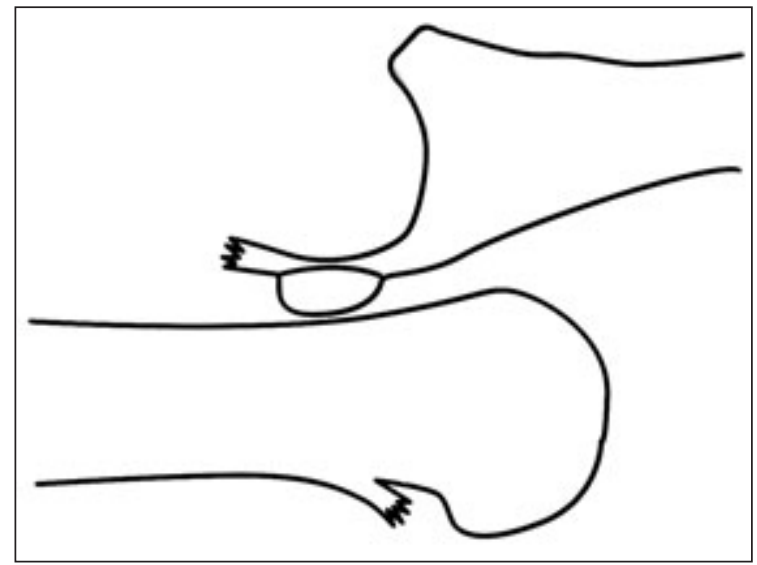

Figura 14: Esquema de la luxación compleja de la MCF del pulgar.

2. Luxación palmar: Es mucho menos frecuente que la dorsal, indicando algunos autores ${ }^{63}$ una proporción de 1/10. El mecanismo es un fuerza desde el dorso cuando la articulación está flexionada. Generalmente la falange se desplaza hacia delante y hacia externo o interno, manteniéndose la falange distal en hiperextensión. Los ligamentos laterales habitualmente no están lesionados y una vez reducida la articulación suele ser estable. El estudio radiográfico nos mostrará la lesión. La reducción la realizaremos mediante una tracción longitudinal sobre la falange. En caso de que tras la reducción apreciáramos una tendencia a la subluxación anterior habría que plantearse la posibilidad de que estuvieran lesionadas las estructuras dorsales (cápsula y aparato extensor) y fuera preciso realizar una reparación quirúrgica. Asimismo se debe valorar la estabilidad lateral ante una posible lesión de los ligamentos laterales. También se han descrito casos de precisar una reducción quirúrgica ante la imposibilidad de lograrla de manera ortopédica ${ }^{66-68}$.

\section{LESIONES IF DEL PULGAR}

Anatómicamente debemos resaltar en la IF la existencia de unos ligamentos colaterales, y sobre todo la inserción de los tendones flexor y extensor, los cuales contribuyen a dar mayor estabilidad a la articulación.

La lesión más frecuente, aunque menos que en la MCF es la luxación, la cual habitualmente es dorsal o lateral, y con cierta frecuencia son abiertas por lesión de la piel.

Habitualmente es fácil lograr una buena reducción mediante tracción longitudinal de la falange y presión en su dorso. Una vez reducida suele ser estable, precisando únicamente una inmovilización con discreta flexión durante 2-3 semanas

Se han descrito casos de irreductibilidad, generalmente por interposición en la articulación de diversas estructuras como pueden ser la placa palmar, un fragmento óseo avulsionado o el tendón flexor pollicis longus ${ }^{69-72}$. En estos casos es necesario el realizar un tratamiento quirúrgico para extraer la estructura interpuesta y reducir la articulación.

En cuanto a las lesiones crónicas de esta articulación la inestabilidad articular es muy rara; generalmente los problemas secundarios vienen determinados sobre todo por la persistencia de una sinovitis dolorosa, la cual puede requerir la 
práctica de una sinovectomía o en casos rebeldes la realización de una artrodesis.

En otros casos en los que se ha desinsertado la placa volar persistiendo una hiperextensión articular pueden requerir la práctica de una tenodesis con el flexor pollicis longus como describieron Eaton y Littler ${ }^{73}$.
También están descritos casos de luxaciones antiguas de la IF no reducidas que se diagnostican al cabo de un tiempo por la falta de flexión de la articulación. En estos casos es necesario el proceder a una reducción quirúrgica debiendo mantener una inmovilización durante cuatro semanas.

\section{BIBLIOGRAFÍA}

1. Gilbert A, Fachinelli A, Kahlit G, et al. Lésions des plaques palmaires. En: Tubiana R (Ed). Traité de Chirurgie de la Main. T2. Paris: Masson, 1984: 783-9.

2. Irisarri $\mathrm{C}$. Lesiones de los ligamentos de los dedos. En: Patología Traumática de la Mano. Madrid: Lab. Cepa, 1992: 7-24.

3. Bento J, Lisón A, Alarcón A. Luxaciones M-F. Particularidades anatómicas y de tratamiento. Rev Esp Cir Mano, 1987; 15: 63-72.

4. Glickel S, Barron OA, Catalano III LW. Luxaciones y lesiones de ligamentos en los dedos. En: Green's Cirugía de la Mano. $5^{\text {a }}$ Edición. Editorial Marban, Madrid, 2007; 343-388.

5. Lourie GM, Gaston RG, Freeland AE. Collateral ligament injuries of the metacarpophalangeal joints of the fingers. Hand Clin, 2006; 22: 357-64.

6. Josty JC, Laing JHE, Den C. Reconstruction of disruption of the deep transverse metacarpal ligament of the ring finger using the A1 pulleys. J Hand Surgery Br, 2006; 31: 439-40.

7. Mauro E. Bloqueo de la MF del dedo medio con subluxación por rotura-interposición de la placa palmar. Rev Esp Cir Mano, 1977; 5: 43-50.

8. Sawant N, Kulikov Y, Giddins GEB. Ooutcome following conservative treatment of metacarpophalangeal collateral ligament avulsion fractures of the finger. J Hand Surg Eur, 2007; 32e: $102-4$

9. Shewring DJ, Thomas RH. Avulsion fractures from the base of the proximal phalanges of the fingers. J Hand Surg $\mathrm{Br}$, 2003; 28: 10-4.
10. Gee TC, Pho RWH. Avulsionfracture at the proximal attachment of the radial collateral ligament of the fifth metacarpophalangeal joint - A case report. J Hand Surg Am, 1982; 7: 526-7.

11. Shewring DJ, Thomas RH: Collateral ligament avulsión fractures from the heads of the metacarpals of the fingers. J Hand Surg Br, 2006; 31: 537-41.

12. Kang L, Rosen A, Potter HG, et al. Rupture of the radial collateral ligament of the index metacarpophalangeal joint: diagnosis and surgical treatment. J Hand Surg Am, 2007; 32: 78994.

13. Gaston RG, Lourie GM. Radial collateral ligament injury of the index metacarpophalangeal joint: an underreported but important injury. J Hand Surg Am, 2006; 31: 1355-61.

14. Green DP, Terry GC. Complex dislocation of the metacarpophalangeal joint. Correlative pathological anatomy. J Bone Joint Surg Am, 1973; 55: 1480-6.

15. Martínez Pagan J, Mena R. Luxación de la articulación MF del quinto dedo. Rev Esp Cir Mano, 1990; 17: 37-40.

16. Mijares JA, Aramburo F. Luxación compleja o irreductible de la metacarpofalangica del índice. Rev Esp Cir Mano, 1978; 6: 67-73.

17. Campuzano A, Fernández Camara J, Garijo P. Luxación abierta de la quinta articulación metacarpo falángica. Rev Esp Cir Mano, 1983; 11: 69-76.

18. Kaplan B. Dorsal dislocation of the metacarpophalangeal joint of the index finger. J Bone Joint Surg Am, 1957; 39: 1081-6.
19. Farabeuf LH. De la luxation du pouce en arrière. Bull Acad Chir, 1876; 2: 21.

20. Murasi T, Moritomo H, Yoshikawa H. Palmar dislocation of the metacarpophalangeal joint of the finger. $\mathrm{J}$ Hand Surg $\mathrm{Br}$, 2004; 29: 90-3.

21. Wood MB, Dobyns JH. Chronic,complex volar dislocation of the metacarpophalangeal joint: report of three cases. J Hand Surg Am, 1981; 5: 73-6.

22. Hargarten SW, Hanel DP. Volar metacarpal phalangeal joint dislocation: a rare and often missed injury. Ann Emerg Med, 1982; 21: 1157-9.

23. Eaton RG. Lésions recentes et anciennes des ligaments des doigts. En: Tubiana R (Ed). Traité de Chirurgie de la Main. t. 2. Paris: Masson, 1984; 751-70.

24. Rodríguez Ferrer E, Grau P, Méndez $\mathrm{J} \mathrm{M}$, et al. Luxación dorsal irreductible articulación IFP dedo medio mano. Mc Medical, 2007; 15: 156-7.

25. Otani K, Fukuda K, Hamanishi C. An unusual dorsal fracturedislocation of the proximal interphalangeal joint. J Hand Surg Eur, 2007; 32: 193-4.

26. Aladin A, Davis TRC. Dorsal fracture dislocation of the proximal interphalangeal joint: A comparative study of percutaneous Kirschner wire fixation versus open reduction and internal fixation. J Hand Surg Br, 2005; 30: 120-8.

27. Lee JY, Teoth LC. Dorsal fracture dislocation of the proximal interphalangeal joint treated by open reduction and interfragmentary screw fixation: Indications, approaches and results. J Hand Surg Br, 2006; 31: 138-46. 
28. Hastings H, Ernst JM. Dynamic external fixation for fractures of the proximal interphalangeal joint. Hand Clinics, 1993; 9: 659-74.

29. Hastings $\mathrm{H}$, Carroll IV C. Treatment of closed articular fractures of the metacarpophalangeal and proximal interphalangeal joints. Hand Clinics, 1988; 4: 503-27.

30. Suzuki Y, Matsunaga T, Sato $S$, et al. The pins and rubber traction system for treatment of comminuted intraarticular fractures and fracture dislocations in the hand. J Hand Surg, 1994; 19: 98-107.

31. Simeonov L, Deliverski T. Fracturas articulares de pequeños huesos en la mano. Diagnóstico y problemas terapéuticos. Rev Esp Cir Mano, 1995; 22: 59-68.

32. Suárez Peña V, Pombo L. Distorsiones interfalangicas de los dedos. Rev Esp Cir Mano, 1977; $5: 87-96$.

33. Aramburo F, Rocamora M. Lesiones capsulares de las articulaciones de los dedos de la mano. Rev Esp Cir Mano, 1979; 7: 89-100.

34. Nanno $M$, Sawaizumi $T$, Ito $H$. Irreducible palmar dislocation of the proximal interphalangeal joint of a finger evaluated by magnetic resonance imaging: a case report. Hand Surg, 2004; 9: 253-6.

35. North E R, Littler JW: Transferring the flexor superficialis tendon: Technical considerations in the prevention of proximal interphalangeal joint disability. J Hand Surg Am, 1980; 5: 498-501

36. Kleinert H, Kasdem ML. Reconstruction of the chronically subluxed proximal interphalangeal finger joint. J Bone Joint Surg Am, 1965; 47: 958-64.

37. Morisawa Y, Ikegami H, Izumida R. Irreductible palmar dislocation of the distal interphalangeal joint. J Hand Surg $\mathrm{Br}$, 2006; 31: 296-7.

38. Bento J, Meseguer LR. Luxación de articulaciones interfa- langicas proximal y distal en un mismo dedo. Rev Esp Cir Mano, 1989; 16: 45-8.

39. Stener B. Entorses récentes de l'articulation métacarpo-phalangienne du pouce. En: Tubiana $\mathrm{R}(\mathrm{Ed})$. Traité de Chirurgie de la Main. t. 2. Paris: Masson. 1984: 770-8.

40. Edelstein DM, Kardashian G, Lee SK. Radial collateral ligament injuries of the thumb. J Hand Surg Am, 2008; 33: 76070.

41. Stener B. Displacement of the ruptured ulnar collateral ligament of the metacarpo-phalangeal joint of the thumb. J Bone Joint Surg Br, 1962; 44: 869-79.

42. Ortiz L, Suárez V. Roturas ligamentosas en la metacarpo falángica del pulgar. Rev Esp Cir Mano, 1985; 13: 115-29.

43. Malik AK, Morris T, Chou D, et al. Clinical testing of ulnar collateral ligament injuries of the thumb. J Hand Surg Eur, 2009; 34: 363-6.

44. Cooper JG, Johnstone AJ, Hider $\mathrm{P}$, et al. Local anaesthetic infiltration increases the accuracy of assessment of ulnar collateral ligament injuries. Emerg Med Australasia, 2005; 7: 1326.

45. Gherissi A, Moussaoui A, Liverneaux $P$. Is the diagnosis of Stener's lesion echographdependent? A series of 25 gamekeeper's thumb. Chir Main, 2008; 27: 216-21.

46. Hahn P, Schmitt R, Kall S. Stener lesion yes or no? Diagnosis by ultrasound. Handchir Mikrochir Plast Chir, 2001; 33: 46-8.

47. Shinohara T, Horii E, Majima $\mathrm{M}$, et al. Sonographic diagnosis of acute injuries of the ulnar collateral ligament of the metacarpophalangeal joint of the thumb. J Clin Ultrasound, 2007; 35: 73-7.

48. Ebrahim FS, De Maeseneer $M$, Jager $T$, et al. US diagnosis of UCL tears of the thumb and Stener lesions: technique, pattern-based approach, and differential diagnosis. Radiographics, 2006; 26: 1007-20.
49. Horch RE, Dragu A, Polykandriotis $E$, et al. Radial collateral ligament repair of the thumb metacarpophalangeal joint using the abductor pollicis brevis tendon. Plast Reconstr Surg, 2006; 117: 491-6.

50. Demirel M, Turhan E, Dereboy $F$, et al. Surgical treatment of skier's thumb injuries: case report and review of the literature. Mt Sinai J Med, 2006; 73: 81821.

51. Katolik LI, Friedrich J, Trumble TE. Repair of acute ulnar collateral ligament injuries of the thumb metacarpophalangeal joint: a retrospective comparison of pull-out sutures and bone anchor techniques. Plast Reconstr Surg, 2008; 122: 1451-6.

52. Catalano LW 3rd, Cardon L, Patenaude $\mathrm{N}$, et al. Results of surgical treatment of acute and chronic grade III [corrected] tears of the radial collateral ligament of the thumb metacarpophalangeal joint. J Hand Surg Am, 2006; 31: 68-75.

53. Campbell CS. Gamekeeper's thumb. J Bone Joint Surg Br, 1955; 37: 148-9.

54. Baskies MA, Tuckman D, Paksima N, et al. A new technique for reconstruction of the ulnar collateral ligament of the thumb. Am J Sports Med, 2007; 35: 1321-5.

55. Neviaser R. Rupture of the ulnar collateral ligament of the thumb (Gameskeeper thumb). $J$ Bone Joint Surg Am, 1971; 53: 1357-64.

56. Fusetti C, Papaloizos M, Meyer $\mathrm{H}$, et al. The ECRL bonetendon ligamentoplasty for chronic ulnar instability of the metacarpophalangeal joint of the thumb. Chir Main, 2005; 24: 217-21.

57. Mitsionis G, Varitimidis SE, Sotereanos GG. Treatment of chronic injuries of the ulnar collateral ligament of the thumb using a free tendon graft and bone suture anchors. J Hand Surg Br, 2000; 25: 208-11. 
58. Glickel SZ, Malerich M, Pearce SM, et al. Ligament replacement for chronic instability of the ulnar collateral ligament of the MP joint of the thumb. J Hand Surg Am, 1993; 18: 93041.

59. Verdan Cl. Traitement chirurgicale des sequelles des entorses MP du pouce. En: Traumatismes osteoarticulaires de la Main. Monographie GEM. Ed L'Expansion. Paris 1971. 115-121

60. Wu WC, Wong TC, Yip TH. Chronic finger joint instability reconstructed with bone-ligament-bone graft from the iliac crest. J Hand Surg Br; 2004; 29: 494-501.

61- Wong TC, Ip FK, Wu WC. Bone-periosteum-bone graft reconstruction for chronic ulnar instability of the metacarpophalangeal joint of the thumb--minimum 5-year follow-up evaluation. J Hand Surg Am, 2009; 34: 304-8.

62. Lee SK, Kubiak EN, Lawler E, et al. Thumb metacarpophalangeal ulnar collateral ligament injuries: a biomechanical simulation study of four static reconstructions. J Hand Surg Am, 2005; 30: 1056-60.

63. Sedel L. Luxations de l'articulation metacarpophalangienne du pouce et des doigts. En: Tubiana R (Ed). Traité de Chirurgie de la Main, t. 2. Paris: Masson. 1984: 783-9.

64. Takami H, Takahashi S, Ando M. Complete dorsal dislocation of the MP joint of the thumb. Arch Orthop Trauma Surg, 1998; 118: 21-24.

65. Hughes LA, Freibereg A. Irreductible MP joint dislocation due to entrapment of FPL. J Hand Surg Br, 1993; 18 (6): 708-9

66. Hirata H, Takegami K, Nagakura T. Irreducible volar subluxacion of the MP joint of the thumb. J Hand Surg Am, 2004; 29: 921-4.

67. Arbelo Rodríguez A, Moya Aparicio $A$, Oliver Roca $G$, et al. Luxación palmar de la MF del pulgar. Rev Ortop Traum, 1991; 35: 384-6.

68. Proubasta I. Volar dislocation of the MP Joint of the thumb: Re- port of two cases. Bull Hosp JT Dis, 1993; 53: 13-4.

69. Kubota R, Nozawa M, Martsuda $\mathrm{K}$, et al. Closed irreductible dislocation of the IP joint of the thumb: Repor of two cases. Chir Organi Mov, 2009; 93: 858.

70. Verhelle W, Von Ransbeeck H, De Swet L. Irreductible dislocation of the IP joint of the thumb: A case report. Eur $\mathrm{J}$ Emerg Med, 2003; 10: 347-8.

71. Gerard F, Pem R, Garbuio P, et al. Irreductible dorsal dislocation of the IP joint of the thumb due to the palmar plate. A case report. Ann Chir Main, 1998; 17: 63-7.

72. Failla JM. Irreductible thumb IP joint dislocation due to a sesamoid and palmar plate: $A$ case report. J Hand Surg Am, 1995; 20: 490-1.

73. Eaton RG. Lésions récentes et anciennes des ligaments des doigts. Lésions de l'articulation interphalangienne du Ponce. En: Tubiana R. Traité de Chirurgie de la Main, t. 2. Paris: Masson, 1984: 763-764 